\title{
Avances en la comprensión de las identidades a partir de las perspectivas de género en espacios militares ${ }^{1}$
}

\author{
https://doi.org/10.21830/9789585380219.01
}

\author{
Maria Antonieta Corcione Nieto ${ }^{2}$ \\ Escuela Militar de Cadetes "General José María Córdova” \\ Leidy Johana Cabrera-Cabrera ${ }^{3}$ \\ Escuela de Aviación del Ejército \\ Andrés Eduardo Fernández-Osorio ${ }^{4}$ \\ Escuela Superior de Guerra "General Rafael Reyes Prieto"
}

Resumen. El objetivo de esta investigación descriptiva analítica es comprender la configuración de la construcción de identidades en el Ejército Nacional desde un enfoque de género, que incluye como ejes de análisis lo femenino-masculino, los roles tradicionales y renovadores, y las identidades colectivas e individuales en el espacio militar. Por medio de la aplicación de una encuesta a mujeres militares del Ejército Nacional, diligenciada por 394 oficiales y 124 suboficiales, se obtuvo como resultado que la configuración de la identidad femenina atraviesa los valores sociales tradicionales que provienen de la conformación familiar, lo cual se refleja en la inscripción a un arma que se considera tradicional

1 Este capítulo forma parte de los resultados del proyecto de investigación "Enfoques diferenciales en el Ejército Nacional: Una propuesta desde las ciencias militares”, del Grupo de Investigación en Ciencias Militares de la Escuela Militar de Cadetes "General José María Córdova”, registrado con el código COL0082556 de Minciencias. Los puntos de vista y los resultados presentados en este capítulo pertenecen a los autores y no reflejan necesariamente los de las instituciones participantes.

$2 \mathrm{PhD}$ en Antropología de la Universidad de los Andes, Colombia. Magíster en Biología Humana de la Universidad Autónoma de Barcelona y la Universidad de Barcelona, España. Antropóloga de la Universidad de los Andes, Colombia. Líder del Grupo de Investigación en Ciencias Militares de la Escuela Militar de Cadetes “General José María Córdova”. OrCid: https://orcid.org/0000-0002-3509-5519 Contacto: maria.corcione@esmic.edu.co

3 Magíster en Docencia e Investigación Universitaria de la Universidad Sergio Arboleda, Colombia y especialista en Docencia e Investigación Universitaria de la misma institución. Trabajadora social de la Universidad de La Salle, Colombia. Directora del Observatorio de Género Militar de la Escuela Militar de Cadetes “General José María Córdova”. Orcid: https://orcid.org/0000-0002-6398-6933 - Contacto: leidy.cabrera@esmic.edu.co

4 Teniente Coronel del Ejército Nacional de Colombia. PhD en Derecho y Ciencia Política de la Universidad de Barcelona, España. Magíster en Economía, Estado y Sociedad: Política y Seguridad de la Universidad Colegio de Londres, Reino Unido. Magíster en Relaciones Internacionales de la Escuela Superior de Economía Moscú, Federación de Rusia. Orcid: https://orcid.org/0000-0003-0643-0258 Contacto: andres.fernandez@buzonejercito.mil.co 
femenina, además de la valoración positiva de movimientos pro vida y la valoración negativa de la libertad sexual. Se concluye que, aunque las mujeres militares se conciben como transgresoras al ingresar al espacio militar, establecen sus identidades en el espectro femenino tradicional como oposición a lo masculino.

Palabras clave: identidad; espacio militar; Fuerzas Militares; perspectiva de género.

\section{Introducción}

Es evidente que un proceso de integración que aspira a construir condiciones de igualdad de derechos y oportunidades en una organización cuya función se ha realizado, históricamente, solo por varones — lo cual ha promovido prácticas, lenguajes y códigos que la hacen institucionalmente de tendencia masculina-, necesita contemplar y revisar cómo se da la dinámica relacional entre hombres y mujeres militares, qué estrategias asumen y cuáles son sus efectos en la vida de los individuos. Estos elementos brindan un horizonte de sentido en la definición de enfoque de género para el Ejército Nacional de Colombia en su búsqueda de equidad. Con esto en mente, la pregunta de investigación de este estudio fue: ¿Cómo se configura la construcción de identidades en el Ejército Nacional desde un enfoque de género?

El análisis del concepto de género para el Ejército Nacional se ha convertido en una tarea permanente que se consolida (Camacho, 2018), entre otros aspectos, en sus diferentes lineamientos normativos. Según se especifica en la Política Pública Sectorial de Transversalización del Enfoque de Género (Ministerio de Defensa Nacional, 2018), este abordaje permite analizar y comprender las características que definen a las mujeres de manera específica, así como sus semejanzas y diferencias, por medio del análisis de las posibilidades vitales de las mujeres, el sentido de sus vidas, sus expectativas y oportunidades, las complejas y diversas relaciones sociales que se dan entre ambos géneros, así como los conflictos institucionales y cotidianos que deben enfrentar a las maneras en que lo hacen (Lagarde, 1996).

A partir de un análisis de las dimensiones constitucionales que se encuentran de manera inicial como marco conceptual: identidad, perspectiva de género y espacio militar - los cuales definen aspectos relevantes desde tres categorías: (1) femenino-masculino, (2) roles tradicionales y renovadores y 
(3) identidades colectivas e individuales_-, se establece en los resultados que el concepto de identidad no es esencialista, sino estratégico y posicional, y repercute sobre el análisis de género. Por esta razón, el abordaje de las identidades configura un asunto multidimensional, esencial en el Ejército Nacional (Corcione \& Cabrera-Cabrera, 2018), de manera que se concluye que la construcción de identidades desde una perspectiva de género en el espacio militar se configura desde lo tradicional.

\section{Identidad}

El proceso de globalización ha generado, por un lado, nuevas identidades como resultado de la apertura de fronteras y, por otro, la reivindicación de lo propio, por parte de ciertos grupos que se resisten a abandonar su cultura (Mercado \& Hernández, 2010, p. 230). Como lo ha expuesto Giménez (1996), la llamada crisis general de identidades afecta a todo el sistema de identidades tradicionales que afrontan el desafío de la modernización.

Desde esta óptica, en la teoría de la identidad social, la identidad se va formando en un proceso dinámico y dialéctico entre el individuo y su situación con un carácter pluridimensional, en donde la pregunta ¿quién soy yo? se responde a partir de la autocategorización en determinados grupos sociales, al mismo tiempo que la autoexclusión — diferenciación- de otros grupos (Bombelli et al., 2013).

En las sociedades modernas, la identidad colectiva no deriva de la coacción, sino que los individuos tienen la opción de seleccionar el grupo que satisfaga sus intereses de manera consciente y voluntaria, con el cual se identifiquen en valores, costumbres, principios, objetivos y simbología (Salazar, 2019). En este sentido, la identidad colectiva no planea sobre los individuos, sino que resulta del modo en que los individuos se relacionan entre sí dentro de un grupo o de un colectivo social. Es decir, es una construcción de sentido social, una construcción simbólica con un ordenamiento evolutivo que guarda formalidades para llegar a nuevas determinaciones (Aguado \& Portal, 1991). Así, una de las características de la identidad colectiva son los rasgos y atributos que de manera participativa forjan los miembros de un grupo desde su interior (Salazar, 2019, p. 113). 
En el caso de las Fuerzas Militares, la identidad colectiva es un sistema abierto, complejo y dinámico que se expresa en el accionar individual y colectivo de sus miembros - lo cual genera vitalidad-, algunos de los cuales evolucionan de acuerdo con el entorno y la necesidad (Salazar, 2019). Esta identidad se ha forjado en los estereotipos masculinos (en contraposición con lo femenino), que se comportan como un marco interpretativo que ha permitido vincular entre sí las experiencias pasadas, presentes y futuras en la unidad de una biografía o de una memoria colectiva (Giménez, 1996). Por ello, la reproducción de sentido parte de diversos espacios y relaciones sociales, y tiende a la multiplicidad en dos dimensiones: (1) multiplicidad de identidades, determinada por factores económicos, políticos, étnicos, y (2) una multiplicidad de niveles de identidad dentro de un mismo grupo reconocido como unidad.

\section{Perspectivas de género}

El concepto de género surge en los años setenta en el contexto de la crítica feminista para explicar, desde una nueva perspectiva, las diferencias entre mujeres y hombres, sobre las cuales se ha justificado a lo largo de la historia la discriminación de unos sobre otros (Méndez, 2007). Así, desde la primera Declaración Universal de Derechos Humanos (1789), filósofos y pensadores se afanaron por justificar la exclusión de las mujeres de los derechos y de la ciudadanía. Para ello argumentaron una diferencia natural, biológica, que asignaba a las mujeres una naturaleza distinta a la de los hombres que explicaba sus menores capacidades para ser plenos sujetos de derechos y decidir sobre sus vidas, excluyéndolas del ámbito de lo público y la política (Méndez, 2007), y por ende de los espacios militares.

La perspectiva o visión de género constituye una categoría analítica que toma los estudios que surgen desde las diferentes vertientes académicas para, desde esa plataforma teórica, cuestionar los estereotipos y elaborar nuevos contenidos que permitan incidir en el imaginario colectivo de una sociedad al servicio de la igualdad y la equidad (Hendel, 2017). El estudio sobre estas perspectivas aborda las diferencias y desigualdades entre hombres y mujeres existentes en la realidad. Es decir, visibiliza el modo en que el género puede afectar la vida y las oportunidades de las personas para resolver sus problemas y dificultades (Méndez, 2007). 
Las ciencias que se han ocupado de avanzar en la comprensión del género destacan tres elementos claves: (1) caracterizaciones sobre las mujeres y sus dinámicas en diferentes escenarios, (2) luchas y retos desde lo femenino y (3) avances en la transversalización del enfoque de género en diferentes niveles de la sociedad. El género es un agente problematizador de dinámicas y transformaciones sociales (Reyes \& Müller, 2016).

Las relaciones de género, lejos de ser dominio exclusivo de una única parcela del conocimiento, o responsabilidad exclusiva de las familias, los educadores o incluso de las políticas de igualdad, son cuestiones de un profundo bagaje sociohistórico y de gran relevancia social (violencia doméstica, explotación infantil, desigualdad laboral, contenidos curriculares e investigaciones sexistas, entre otros). De este modo, se entiende el género y sus relaciones como parte indispensable de los recursos de socialización disponibles para pensar y actuar sobre nosotros mismos y los demás (Villuendas \& Gordo, 2003).

La inclusión de conceptos como el de transversalidad de género (sinónimo de enfoque integrado de género) es una práctica deseable para el avance hacia la igualdad y la equidad real de quienes ejercen los poderes públicos de todas las áreas del Estado (Hendel, 2017). Transversalizar la perspectiva de género es el proceso de valorar las implicaciones que tiene para los hombres y para las mujeres cualquier acción que se planifique, ya se trate de legislación, políticas o programas, en todas las áreas y en todos los niveles. Es una estrategia para conseguir que las preocupaciones y las experiencias de las mujeres, al igual que las de los hombres, sean parte integrante en la elaboración, puesta en marcha, control y evaluación de las políticas y de los programas en todas las esferas políticas, económicas y sociales (Comisión Económica para América Latina y el Caribe [Cepal], 2017).

\section{Espacio militar}

El concepto de espacio se comprende como una construcción social. Desde esta perspectiva, el espacio fisico incluye en su complejidad la creación de un espacio cultural que se finca en lo físico, pero que no se reduce a él.

Para fines prácticos y de manera habitual no se distingue un espacio físico del significado que este tiene para el observador común, sino que tal significado 
resalta solo por contraste entre dos culturas. Por esto, hablar de espacio cultural es hablar de prácticas culturales (formas de consumo, de relación social, de transformación, etc.). Es importante destacarlo porque, para cualquier grupo social, el sentido es un proceso permanente, conformado por prácticas. En este contexto, se entiende como espacio la red de vínculos de significación que se establece en el interior de los grupos, con las personas y las cosas. Dicho de otra manera, el espacio comprende las relaciones proxémicas (de persona a persona) y cósicas (de personas con objetos), siempre comprendidas dentro del ámbito de la significación cultural de un grupo (Aguado \& Portal, 1991, p. 31).

Siguiendo este enfoque, el espacio militar permite una comprensión del país en la medida en que los alcances de las instituciones castrenses inciden sobre las dinámicas sociales porque se construyen en ellas. Este complejo como escenario mantiene principios de fundamentación propios de la institución, que parten de la construcción histórica y de memoria que establecen sus miembros para configurarse como miembros de esta por medio de discursos de construcción identitaria.

Con la inclusión de mujeres en la carrera militar, el espacio de análisis sobre género en escenarios militares se ha venido consolidando de forma estratégica. En consecuencia, resulta de vital importancia comprender el trabajo para democratizar la profesión militar, que avanza en términos de incorporación, pero que debe incluir la transversalización del enfoque de género, eje en el que está el mayor reto en su consolidación en el Ejército Nacional.

\section{Metodología}

Este trabajo presenta un estudio descriptivo analítico de tipo cualitativo para establecer los avances en la comprensión de las identidades en el Ejército Nacional a partir de las perspectivas de género en el contexto militar, las cuales son abordadas mediante un análisis transversal de las categorías femenino-masculino, roles tradicionales y renovadores e identidades colectivas e individuales. Específicamente, se desarrollaron dos categorías fundamentales: las identidades y el enfoque de género, vistos desde las percepciones de mujeres oficiales de arma vinculadas al Ejército Nacional. Los instrumentos empleados para 
esta investigación incluyeron la revisión documental y el desarrollo de una encuesta sobre caracterización de las mujeres militares del Ejército Nacional diligenciada por 394 (76 \%) oficiales y 124 (24\%) suboficiales de la institución. Asimismo, se realizaron pruebas de Chi2 en el programa estadístico SPSS versión 26.

\section{Resultados}

Los resultados indican que el $92,4 \%$ de las mujeres oficiales y suboficiales encuestadas pertenecen al cuerpo de las armas y el 7,6 \% pertenecen al cuerpo administrativo, distribuidas de la siguiente manera en las diferentes armas del Ejército Nacional: la mayoría de las mujeres pertenecen al arma de Logística (35,3\%), mientras que en las armas de Infantería (3,3\%) y Aviación del Ejército (2,3\%) tienen un menor porcentaje de participación (figura 1).

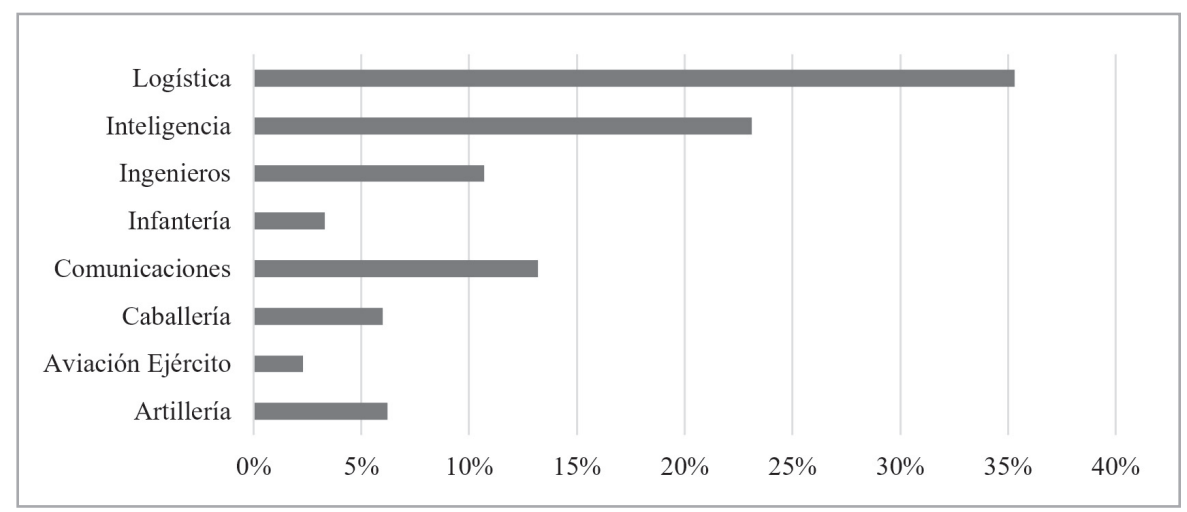

Figura 1. Distribución de mujeres militares en las armas del Ejército Nacional.

Fuente: Elaboración propia.

De manera inicial, se puede resaltar que la pertenencia de las mujeres en las diferentes armas refleja la inscripción de los roles que cumplen las mujeres dentro del Ejército Nacional, lo cual se refuerza en la limitación de entrada a ciertas armas que se consideran no aptas para mujeres. Aunque el ingreso a la institución castrense por parte de las mujeres se asume como una transgresión a los roles femeninos-masculinos inscritos en la discusión de lo tradicional 
renovador, la inscripción a las armas establece un patrón tradicionalista en los roles que puede asumir una mujer en los escenarios militares.

Dentro de este paradigma de la constitución de una identidad de lo femenino como mujer militar, es preciso comprender los aspectos que configuran esa identidad como colectivo de un nosotras. Por ello se realizaron preguntas sobre libertades de derechos y derechos fundamentales que afectan directamente a las mujeres. Se encuentra que son altos los niveles de indiferencia frente a la prostitución $(29,5 \%)$ y a la libertad sexual $(22,3 \%)$, mientras que la aprobación de la eutanasia $(63,3 \%)$ y la pena de muerte $(71,9 \%)$ superan más de la mitad de la muestra (figura 2). Por el contrario, la prostitución es el aspecto en el que se presenta la mayor desaprobación por parte de las mujeres militares, con el 66,5\% de desacuerdo (figura 2).

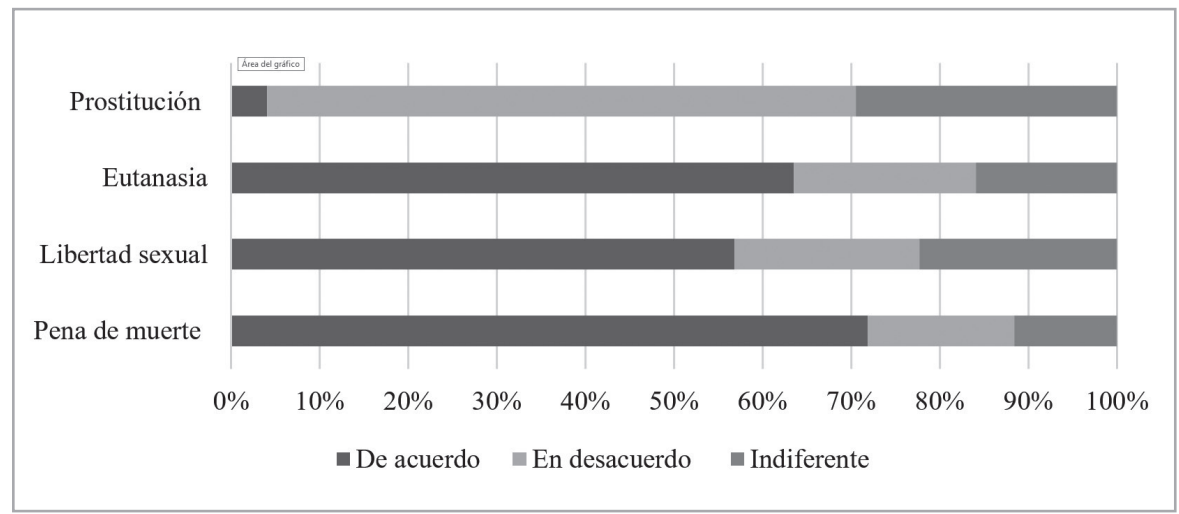

Figura 2. Niveles de aprobación sobre libertad de derechos y derechos fundamentales. Fuente: Elaboración propia.

No se halló asociación estadísticamente significativa entre la pertenencia al cuerpo de armas y el administrativo cuando se comparan con la percepción de las mujeres encuestadas respecto a las libertades de derechos y los derechos fundamentales. Asimismo, no se encontraron diferencias estadísticamente significativas entre las creencias religiosas (católica en el 86,8 \% de las encuestadas) y las libertades de derechos y derechos fundamentales, lo cual indica que las variables son independientes. En el caso de la relación entre la aprobación de la pena de muerte y el tipo de familia (tradicional o moderna), los resultados 
muestran que la asociación estadística no es significativa. No obstante, sí existe asociación estadísticamente significativa entre los valores de desaprobación de la libertad sexual y la familia tradicional $\left(X^{2}=23,940 p=0,000\right)$.

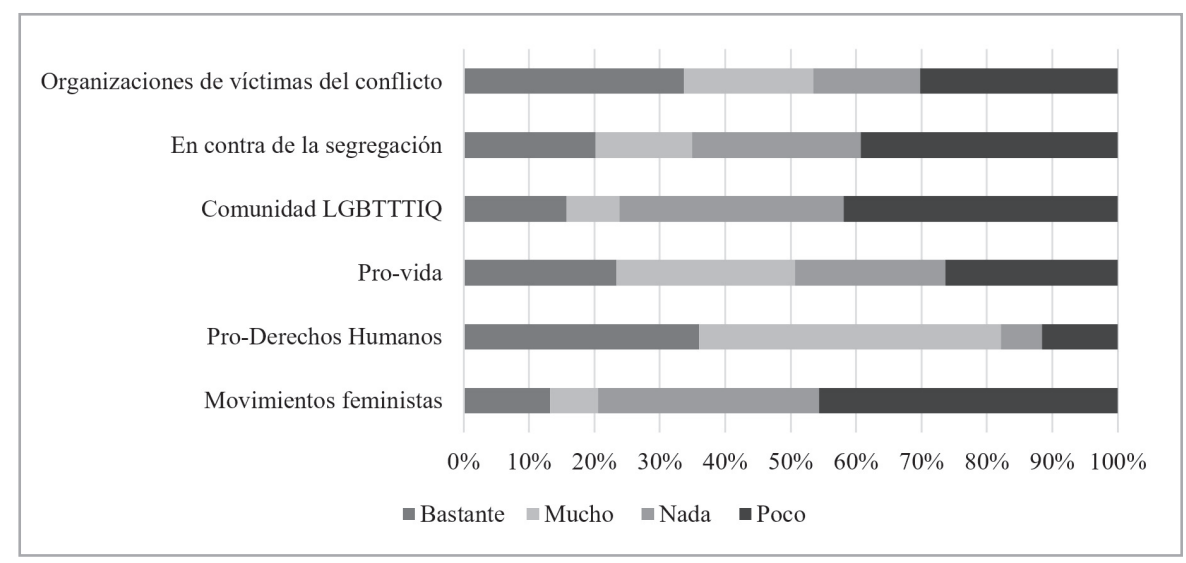

Figura 3. Apoyo a organizaciones y movimientos sociales.

Fuente: Elaboración propia.

Para el caso del apoyo a las organizaciones y movimientos sociales, el $33,7 \%$ de las encuestadas apoya bastante a las organizaciones de víctimas del conflicto y a las organizaciones pro Derechos Humanos (36\%) (figura 3), temas que están vinculados directamente con su práctica militar. Aun cuando no existe una relación de dependencia entre las variables movimientos pro vida y el ambiente religioso en su casa, se encuentra una ligera asociación positiva en ambientes bastante religiosos con el apoyo a los movimientos pro vida. A pesar de que los resultados no muestran una asociación estadísticamente significativa entre la percepción sobre los movimientos feministas y el tipo de familia, sí se encuentra una asociación positiva entre el poco apoyo a los movimientos feministas y la familia tradicional. Por último, la única dependencia estadísticamente significativa se encuentra entre el poco apoyo a la comunidad LGBTIQ y pertenecer a una familia tradicional $\left(X^{2}=25,255 p=0,000\right)$. 


\section{Discusión}

Los resultados de este estudio presentan varios retos en la comprensión de las dinámicas identitarias desde un enfoque de género, debido al carácter dinámico y posicional de estas en las relaciones entre hombres y mujeres del Ejército Nacional. El problema no es el estudio de las diferencias en sí; la problemática es que se permita transformar las miradas y la capacidad crítica sobre los objetos de estudio, lo cual hace evidente la necesidad de multiplicar las voces en el análisis y las interpretaciones de los problemas compartidos que no escapan del ámbito militar.

Los retos en el análisis de la identidad consisten en su carácter posicional y la multiplicidad de identidades, configurada por factores económicos, políticos, sociales y culturales, así como en los niveles de identidad dentro de un mismo grupo reconocido como unidad. Este último aspecto determina la manera como se asume un género (en este caso el femenino) en el Ejército Nacional. De acuerdo con los resultados, las identidades de las mujeres militares se construyen en la dicotomía de lo masculino y lo femenino estereotípico, y específicamente este último como un espacio conservador.

Este espacio conservador se refuerza al momento de contemplar las posiciones sobre las libertades de derechos y los derechos fundamentales que las afectan como grupo identitario femenino. En este caso, se refuerzan los sistemas de valores tradicionales, que las unifica y permite verlas como un grupo unitario muy conservador (sin distinción entre mujeres suboficiales y oficiales). Por el contrario, al pensar en el apoyo a los movimientos de víctimas y pro Derechos Humanos, se desligan de su identidad femenina conservadora y se vinculan a una identidad unificada militar desde la construcción simbólica del servicio. Con ello, pasan a situarse en roles renovadores.

Por su parte, los retos en servirse del enfoque de género se observan en el momento de desligar las posibles explicaciones de las dinámicas relacionales de la identidad, para este caso la femenina, de las explicaciones contextuales de las mujeres. Asumir que la identidad colectiva permea y diluye toda multiplicidad del proceso identitario individual en la carrera militar es un error. De allí la importancia de comprender la constitución de las experiencias personales y 
familiares, que para el caso de estudio resultan ser decisivas sobre la configuración de la participación en un arma. La pertenencia a familias tradicionales, al igual que las creencias religiosas católicas, configuran mujeres que, aunque asumen un papel renovador al ingresar al Ejército Nacional, en la elección de una carrera en armas transitan durante su carrera militar en roles tradicionales dentro de la configuración del espacio militar.

Este trabajo inicial no permite profundizar en el entendimiento de los tránsitos en la configuración de lo identitario femenino militar, por lo cual se asume de forma preliminar que la misma configuración de la identidad es algo transformador y dialéctico con un carácter pluridimensional, casi camaleónico.

La transversalización del enfoque de género, por tanto, permite que se conjuguen nuevas perspectivas de análisis para la comprensión de las relaciones sociales. A pesar de la importancia de los logros de la visión diferencial en la investigación de las problemáticas propias de las dinámicas sociales, en el campo de las ciencias militares los debates han sido poco desarrollados y se han centrado en las caracterizaciones poblacionales del personal del Ejército Nacional.

Siendo así, la apropiación de espacios militares enfrenta el reto de integrar a las mujeres y a los hombres para construir condiciones de igualdad de derechos y oportunidades de manera plena en el Ejército Nacional. Como institución con determinadas características que se repiten en diversos contextos temporales y culturales, el Ejército Nacional es el producto de un proceso histórico que se cristaliza en una organización que no solo tiene importantes capacidades materiales, sino también importantes componentes valorativos y connotaciones simbólicas que contribuyen a su funcionamiento interno y a la producción de sentido en el campo social. Concebir el espacio militar como un espacio vivo, activo y por lo tanto social permite vislumbrar la transformación institucional del Ejército Nacional.

\section{Conclusiones}

Con un escenario poco explorado de estudios sociales de lo militar en Colombia, el desarrollo de las nuevas reflexiones sobre las dinámicas sociales se 
hace pertinente. De acuerdo con lo señalado, se puede concluir en esta investigación que la configuración de la construcción de identidades en el Ejército Nacional de las mujeres oficiales y suboficiales se encuentra mediada por la dinámica de roles opuestos de lo masculino y lo femenino. Desde una perspectiva de género se ha establecido que, aunque exista la transgresión identitaria de lo femenino al ingresar al Ejército Nacional, una vez en este espacio militar, las mujeres se integran a espacios más tradicionales.

Ante esta situación de configuración identitaria y por medio del análisis de las percepciones ante movimientos y organizaciones sociales, derechos fundamentales y libertades de derecho, se estableció que las mujeres pertenecientes al Ejército Nacional tienden a ser más tradicionales. La pertenencia a familias tradicionales y de creencia religiosa católica refuerza en ellas su posición tradicionalista y conservadora, entendida como aquellas que guardan, protegen y respetan las tradiciones.

Resulta primordial seguir en el camino del entendimiento de las mujeres que componen los espacios militares en el Ejército Nacional desde el análisis de la construcción de identidades. Lo expuesto a lo largo de este trabajo permite plantear que, desde una perspectiva de género, se podrá avanzar en la comprensión de las dinámicas entre hombres y mujeres del Ejército Nacional.

\section{Referencias}

Aguado, J. C., \& Portal, M. A. (1991). Tiempo, espacio e identidad social. Alteridades, 1(2), 31-41. https://alteridades.izt.uam.mx/index.php/Alte

Bombelli, J. I., Sosa, F. M., Fernández, O. D., Cejas, L. A., \& Torres, J. A. (2013, septiembre 20-30). Memoria colectiva: Eventos relevantes de la historia universal para una muestra de población militar [Ponencia]. V Congreso Internacional de Investigación y Práctica Profesional en Psicología: XX Jornadas de Investigación Noveno Encuentro de Investigadores en Psicología del MERCOSUR, Facultad de Psicología, Universidad de Buenos Aires, Argentina. http://jimemorias.psi.uba.ar/index.aspx?anio=2013

Camacho Zambrano, C. (2018). Caleidoscopio: Estudios sobre feminidades y perspectiva de género en el contexto militar colombiano. Sello Editorial ESMIC. https://doi. org/10.21830/9789585200838

Comisión Económica para América Latina y el Caribe [CEPAL]. (2017). Planes de igualdad de género en América Latina y el Caribe: Mapas de ruta para el desarrollo. Naciones Unidas. 
https://www.cepal.org/sites/default/files/events/files/planes_de_igualdad_de_genero_en_ america_latina_y_el_caribe._mapas_de_ruta_para_el_desarrollo.pdf

Corcione Nieto, M., \& Cabrera-Cabrera, L. (2018). Identidad e ideología: Dinámicas culturales entre los estudiantes de la Escuela Militar de Cadetes. En A. Fernández-Osorio \& E. Latorre Rojas (Eds.), La construcción del rol de la mujer militar (pp. 41-66). Sello Editorial ESMIC. https://doi.org/10.21830/9789585692831

Giménez, G. (1996). La identidad social o el retorno del sujeto en sociología. En Identidad: Análisis y teoría, simbolismo, sociedades complejas, nacionalismo y etnicidad. III Coloquio Paul Kirchhoff. Universidad Nacional Autónoma de México.

Hendel, L. (2017). Perspectiva de género: Comunicación, infancia y adolescencia. Guías para periodistas. Fondo de las Naciones Unidas para la Infancia. https://www.unicef.org/argentina/sites/unicef.org.argentina/files/2018-04/COM-1_PerspectivaGenero_WEB.pdf

Lagarde, M. (1996). Género y feminismo: Desarrollo humano y democracia. Horas y Horas.

Méndez, L. (2007). Antropología feminista. Síntesis.

Mercado Maldonado, A., \& Hernández Oliva, A. (2010). El proceso de construcción de la identidad colectiva. Convergencia, Revista de Ciencias Sociales, (53), 229-251. https:// www.redalyc.org/pdf/105/10513135010.pdf

Ministerio de Defensa Nacional. (2018). Politica pública sectorial de transversalización del enfoque de género para el personal uniformado de la Fuerza Pública (2018-2027). https:/www.justiciamilitar.gov.co/irj/go/km/docs/Mindefensa/Documentos/descargas/ Prensa/Documentos/politica_genero.pdf

Salazar, J. C. (2019). Rol de la identidad colectiva de las Fuerzas Militares en el proceso de construcción de la memoria histórica. En E. Pastrana Buelvas \& H. Gehring (Eds.), Fuerzas Militares de Colombia: Nuevos roles y desafios nacionales e internacionales (pp. 109-142). Fundación Konrad Adenauer. https://www.kas.de/documents/287914/287963/ Fuerzas+Militares.pdf/8acb9e5d-ea94-1a12-0f70-23402a752227?t=1555002345379

Reyes, M. I., \& Müller, W. (2016). Psicología y género, nuevos desafíos. Revista de Psicología, 25(2), 1-4. https://scielo.conicyt.cl/pdf/revpsicol/v25n2/art05.pdf

Villuendas, M. D., \& Gordo López, A. (2003). Relaciones de género en psicología y educación. Comunidad de Madrid y Consejería de Educación. http://www.madrid.org/bvirtual/BVCM001401.pdf 